\title{
Neuroprotection and Functional Recovery Associated with Decreased Microglial Activation Following Selective Activation of mGluR2/3 Receptors in a Rodent Model of Parkinson's Disease
}

\author{
Hugh Chan, ${ }^{1}$ Helen Paur, ${ }^{1}$ Anthony C. Vernon, ${ }^{1,2}$ Virginia Zabarsky, ${ }^{1}$ Krishna P. Datla, ${ }^{1}$ \\ Martin J. Croucher, ${ }^{1}$ and David T. Dexter ${ }^{1}$ \\ ${ }^{1}$ Parkinson's Disease Research Group, Faculty of Medicine, Imperial College London, 4th Floor, Burlington Danes Building, \\ Hammersmith Hospital, Du Cane Road, W12 0NN London, UK \\ ${ }^{2}$ Centre for the Cellular Basis of Behaviour, Department of Neuroscience, Institute of Psychiatry, Kings College London, 1st Floor, \\ The James Black Centre, Coldharbour Lane, SE5 9NU London, UK
}

Correspondence should be addressed to David T. Dexter, d.dexter@imperial.ac.uk

Received 16 January 2010; Revised 24 February 2010; Accepted 8 March 2010

Academic Editor: Antonio Pisani

Copyright () 2010 Hugh Chan et al. This is an open access article distributed under the Creative Commons Attribution License, which permits unrestricted use, distribution, and reproduction in any medium, provided the original work is properly cited.

\begin{abstract}
Clinical trials have demonstrated positive proof of efficacy of dual metabotropic glutamate receptor $2 / 3$ (mGluR2/3) agonists in both anxiety and schizophrenia. Importantly, evidence suggests that these drugs may also be neuroprotective against glutamate excitotoxicity, implicated in the pathogenesis of Parkinson's disease (PD). However, whether this neuroprotection also translates into functional recovery is unclear. In the current study, we examined the neuroprotective efficacy of the dual mGluR2/3 agonist, $2 R, 4 R$-4-aminopyrrolidine-2,4-dicarboxylate $(2 R, 4 R$-APDC), and whether this is accompanied by behavioral recovery in a rodent 6-hydroxydopamine (6-OHDA) model of PD. We now report that delayed post lesion treatment with $2 R, 4 R$-APDC (10 nmol), results in robust neuroprotection of the nigrostriatal system, which translated into functional recovery as measured by improved forelimb use asymmetry and reduced $(+)$-amphetamine-induced rotation compared to vehicle treated animals. Interestingly, these beneficial effects were associated with a decrease in microglial markers in the SNc, which may suggest an antiinflammatory action of this drug.
\end{abstract}

\section{Introduction}

Dopamine replacement therapy provides effective relief of motor symptoms in PD patients, but has no proven effect on the degeneration of the nigrostriatal system $[1,2]$. Pathophysiological evidence suggests that neurodegeneration in PD has an excitotoxic component, mediated through hyperactivity of glutamatergic subthalamic nucleus (STN) output pathways as a consequence of striatal dopamine (DA) depletion [3]. Owing to their differential expression and neuromodulatory role in glutamate neurotransmission in the basal ganglia, metabotropic glutamate receptors (mGluR) may represent promising drug targets for PD pharmacotherapy $[4,5]$. Theoretically, mGluR are perceived to be advantageous due to the lack of adverse effects that are induced by ionotropic receptor antagonists [6]. This is borne out by recent positive proof of efficacy of Group II mGluR (comprising mGluR2 and mGluR3 subtypes) agonists in clinical trials of both schizophrenia [7] and generalized anxiety disorder [8], in which these drugs were well tolerated with minimal extrapyramidal side effects. Interestingly, group II mGluR are considered promising drug targets for "neuroprotective receptors", since their activation inhibits glutamate release at key synapses in the basal ganglia, including subthalamonigral synapses that are overactive as a result of striatal DA depletion in PD [9-11]. Further, activation of mGluR2/3 stimulates the production and release of neurotrophic factors from glial cells in vitro $[12,13]$ and in vivo [14-16]. Results from our laboratory and others reveal a moderate neuroprotective effect of Group II mGluR agonists in rodent models of PD, the magnitude of which was dependent on lesion severity $[14,17-19]$. 
Nevertheless, it remains unclear whether these neuroprotective effects also translate effectively into functional recovery in vivo. Indeed, systemic administration of selective dual mGluR2/3 agonists reverses reserpine-induced akinesia, but not rotational asymmetry following amphetamine challenge in vivo, despite concomitant, albeit weak, neuroprotection of the nigrostriatal system [18]. By contrast, other studies have reported reversal of both haloperidol-induced muscle rigidity and catalepsy in rats following Group II mGluR agonist treatment $[9,20]$. On the other hand, intrastriatal injection of the dual mGluR2/3 agonist $2 R, 4 R$-4-aminopyrrolidine2,4 -dicarboxylate $(2 R, 4 R$-APDC) had no effect in these behavioural tests [21]. Interestingly, intranigral injection of the nonselective mGluR2/3 agonist DCG-IV alleviated reserpine-induced akinesia in rodents [22]. However, this compound is not selective for mGluR2/3 receptors alone and thus is not appropriate for use with in vivo models [23].

Taken together, pharmacotherapy targeting Group II mGluR in the basal ganglia appears to offer the potential to combine both symptomatic improvements with potential neuroprotection of remaining nigral neurons $[4,5]$. Nevertheless, there seems to be conflicting data in the extant literature regarding both the degree of neuroprotection and the behavioural effects of dual mGluR $2 / 3$ treatment in vivo in animal models of $\mathrm{PD}$, which may in part be related to the differing routes of administration employed in each study. Thus, our goal in the current study was to extend our initial findings with the Group II mGluR agonist $2 R, 4 R$-APDC to address this question in a rodent partial lesion model of PD. In addition, taking into account the published effects of Group II mGluR activation on glial cells, we have examined inflammatory markers, alongside markers of neuronal loss in the nigra to determine whether intranigral treatment with $2 R, 4 R$-APDC also influences inflammatory changes in vivo.

\section{Experimental Procedures}

2.1. Materials. 2R,4R-APDC was obtained from Tocris Cookson Bioscience (Bristol, UK). All other reagents and compounds were obtained from Sigma-Aldrich Ltd. (Poole, UK) unless stated otherwise in the text.

2.2. Experimental Animals. Male Sprague-Dawley rats (250 \pm 20 g; Harlan UK Ltd., Bicester, UK) were housed in groups of three at $21 \pm 1^{\circ} \mathrm{C}$ on a 12-hour light : dark cycle (lights on 07:00 h, lights off 19:00 h). Standard rat chow and drinking water were available ad libitum throughout the study. All animal experiments were carried out in accordance with the guidelines published in the Home Office Animals (Scientific Procedures) Act, UK, 1986 under project license number PIL 70/6486, with local ethical approval (Ethical Committee of Imperial College, London).

2.3. Stereotaxic Implantation of Guide Cannulae. Rats were anaesthetized with a mixture of isoflurane $(5 \%$ for induction, $1 \%-3 \%$ for maintenance, flow rate $1 \mathrm{~L} / \mathrm{min}$ ) in medical air/oxygen mixture $(70 / 30 \%)$ and placed in a stereotaxic frame (David Kopf instruments, Tujunga, USA), with the incisor bar set at $3.3 \mathrm{~mm}$ above the interaural line [24]. Body temperature was monitored using a rectal probe and maintained at $37^{\circ} \mathrm{C}$ during surgery using a thermostatically controlled heated mat. Stainless steel guide cannulae (26gauge, Plastics One, Roanoke, VA, USA) were unilaterally implanted $1 \mathrm{~mm}$ above the left $\mathrm{SNc}$ at the following coordinates; AP: $-3.0 \mathrm{~mm}$ and ML: $+2.5 \mathrm{~mm}$ (relative to bregma), DV: $-7.6 \mathrm{~mm}$ (relative to dura; Paxinos and Watson, 1986) as described previously [19]. Postsurgery, rats were placed in a heated recovery chamber to recover from the anaesthetic. Postoperative care included individual caging, analgesia (buprenorphine, $0.3 \mathrm{mg} / \mathrm{kg}$ s.c during the first 48 hours), fluid replacement ( $4 \mathrm{~mL} 0.18 \%$ glucosaline solution i.p), and mashed high-nutrient food pellets during the first week after surgery. Animals were checked daily for signs of gross neurological or behavioural abnormalities and weighed to monitor recovery. No experimental interventions were made until animals had completed a minimum 10-day recovery period.

2.4. Experimental Design. Following recovery from surgery, cannulated animals were randomly assigned to drug $(n=8)$ or vehicle treatment groups $(n=10)$. To investigate the effects of delayed 2R,4R-APDC-treatment on neuroprotection, 6OHDA lesioned animals received intranigral injections of $10 \mathrm{nmol} 2 R, 4 R$-APDC or drug vehicle, once daily, for seven consecutive days as per our previously published protocol [19] with the exception that drug treatment was delayed until 48 hours post lesion. To evaluate whether $2 R, 4 R$ APDC improved the behavior of 6-OHDA lesioned animals, both treated and control animals were assessed for their performance in the spontaneous forelimb test and the degree of contralateral rotation following amphetamine injection. Performance in the forelimb asymmetry test was assessed prior to lesioning and drug treatment to establish a baseline and control for the effects of cannulae implantation on behavior. Animals were retested for performance in this test upon completion of drug treatment (7 days), equivalent to 9 days post lesioning. Animals were assessed for amphetamine-induced rotational behavior 24 hours later. In both treatment groups, neuroprotection was quantified by postmortem immunohistochemical analysis of tyrosine hydroxylase (TH)-positive cells in the substantia nigra pars compacta $(\mathrm{SNpc})$ and biochemical measurement of strital monoamine concentrations by HPLC. In addition neuronal cell counts were performed in the $\mathrm{SNc}$ to control for changes in $\mathrm{TH}$ expression in atrophic neurons. Lastly, the effects of 6-OHDA lesioning and drug or vehicle treatment on inflammatory markers were assessed using postmortem immunohistochemistry for astroglial and microglial cell markers, respectively. Details of 6-OHDA lesioning, drug preparation and administration, behavioral testing, and postmortem immunohistochemical, and biochemical analyses are provided in the sections below.

2.5. Induction of 6-OHDA Nigrostriatal Lesions. Unilateral 6-OHDA nigrostriatal lesions were created as previously described elsewhere [25]. Briefly, $12 \mu \mathrm{g}$ 6-hydroxydopmine 
hydrobromide (6-OHDA, free base, Sigma-Aldrich Ltd. Poole, UK) in $4 \mu \mathrm{L} 0.1 \%$ ascorbic acid/saline solution was infused into the left SNc using a 26-gauge stainless steel injection cannulae, extending $1 \mathrm{~mm}$ below the tip of the indwelling guide cannula, attached with flexible tubing (Portex, Hythe, UK) to a $10 \mu \mathrm{L} 700$ series Hamilton syringe mounted on a motorised Harvard micropump (Harvard Apparatus, Edenbridge, UK). Infusions were made at a rate of $1 \mu \mathrm{L} / \mathrm{min}$ followed by a 5 -minute equilibration time, during which the needle remained in place and was then slowly retracted.

2.6. Drug Preparation and Intranigral Administration. Stock solutions of $2 R, 4 R$-APDC were prepared in phosphate buffered saline (PBS; mM: $\mathrm{NaCl} 137 ; \mathrm{KCl} 2.7 ; \mathrm{KH}_{2} \mathrm{PO}_{4} 1.8$; $\mathrm{Na}_{2} \mathrm{HPO}_{4} 10$ ) and adjusted to $\mathrm{pH}$ 7.4. Drug concentrations were selected based on published in vitro $\mathrm{EC}_{50}$ and $K_{i}$ values for each compound at mGluR2/3 $[23,26]$ and on our previous experience with this compound [19] to avoid confounding effects due to activation of other mGluR subtypes. $2 R, 4 R$-APDC was chosen for use as it retains selectivity for $\mathrm{mGluR} 2 / 3$ even at relatively high concentrations [26]. Drugs were aliquoted and stored at $-80^{\circ} \mathrm{C}$ according to the manufacturer's instructions. Fresh drug aliquots were used on each day of drug administration to avoid repeated freezethaw cycles. In all treatment groups, unilateral intranigral drug injections were performed exactly as described for 6-OHDA lesions, in a final injection volume of $4 \mu \mathrm{L}$. Importantly, in our previous study with this compound [19], drug treatment was initiated one hour prior to 6-OHDA lesioning, which whilst providing robust proof-of-concept, is not ideal. To address this limitation, in the current study drug treatment initiation was delayed until 48 hours post lesion, at which point nigrostriatal degeneration should be ongoing in vivo. In all groups, drug or vehicle treatment was initiated 48 hours post 6-OHDA lesioning. Intranigral injections were performed once daily, at the same time, over 7 consecutive days. Following all intranigral injections, animals were observed qualitatively over a period of 20 minutes for any signs of gross behavioral abnormalities in response to the drug injection.

\subsection{Assessment of Spontaneous Forelimb Use and Rotational} Asymmetry Following (+)-Amphetamine Challenge. Performance in the spontaneous exploratory forelimb use test was assessed as previously described by others $[27,28]$. In brief, animals were placed in a clear plastic cylinder (height: $30 \mathrm{~cm}$, width: $20 \mathrm{~cm}$ ) and forelimb use asymmetry was assessed during rearing behaviour [27]. Forelimb contact with the cylinder wall was scored as left, right, or both and the percentage forelimb asymmetry was determined using the following formula: ((contralateral limb placements ipsilateral limb placements)/total placements) $\times$ 100) [27]. As described above, forelimb asymmetry was assessed 10 days after cannulation and 1 day prior to 6-OHDA lesioning. Animals were then retested prior to sacrifice, following 7 days of drug treatment, equivalent to 9 days post lesion, by an investigator blinded to the treatment group.
Rotational asymmetry was assessed 24 hours after completion of drug treatment, equivalent to 10 days post lesion. Animals were administered amphetamine $(5 \mathrm{mg} / \mathrm{kg}$ i.p.) to induce rotational behavior as described previously [29]. Animals were placed in a clear circular test arena $(25 \times$ $15 \mathrm{~cm}$ ) and following a short period of acclimatization (30 minutes), injected with the dopaminergic agonist. The complete number of contralateral and ipsilateral turns was then recorded for 60 minutes postinjection.

2.8. Tissue Preparation. Upon completion of behavioral testing, 9 days after the initial drug injection and 11 days post lesion, animals were sacrificed by decapitation and the brains quickly dissected out onto a chilled platform and cut at the level of the infundibular stem $(-4.16 \mathrm{~mm}$ from bregma) to produce a fore- and hindbrain block containing the corpus striatum and SNc, respectively. Hindbrain blocks were fixed in $4 \%$ paraformaldehyde for 5 days, cryoprotected in $30 \%$ sucrose for $24-48$ hours and stored desiccated at $-80^{\circ} \mathrm{C}$ until cryostat sectioning. Coronal sections $(20 \mu \mathrm{m}$-thick) were cut on a cryostat, (Bright Instruments, Cambridge, UK), throughout the rostral to caudal extent of the SNc $(-4.80$ to $6.30 \mathrm{~mm}$ from bregma; Paxinos and Watson, 1986). Freefloating sections were collected in series and stored in PBS ( $\mathrm{pH}$ 7.4) containing $0.05 \%$ sodium azide as a preservative until immunostaining. From the forebrain blocks, the left and right striata were quickly dissected out, snap-frozen, and stored at $-80^{\circ} \mathrm{C}$ until biochemical analysis of monoamine content.

2.9. Immunohistochemistry. Immunohistochemistry was performed using a standard immunoperoxidase method as previously described [25]. Briefly, free-floating sections were washed in PBS ( $\mathrm{pH} 7.4,3 \times 5$ minutes $)$ and endogenous peroxidase activity quenched by incubation in $1 \%$ hydrogen peroxide solution (30 minutes) followed by cell permeabilisation in PBS containing $0.1 \%$ triton X-100 (15 minutes). Nonspecific biding was blocked by incubating sections in 3\% normal goat serum (NGS, MP Biomedicals, Eschwage, Germany) diluted in PBS for 1 hour. Sections were incubated with primary antibody diluted in PBS containing 0.1\% triton and 3\% NGS for 18 hours at room temperature. Dopaminergic neurons were identified using rabbit antirat tyrosine hydroxylase (TH, AB151, Chemicon Europe, Watford, UK, $1: 3000$ ) with adjacent sections probed with mouse antirat neuron specific nuclear protein (NeuN, MAB377, Chemicon Europe, $1: 2000$;) to control for changes in $\mathrm{TH}$ protein expression. Astrocytes were identified using rabbit antirat glial fibrillary acidic protein (GFAP, AB5804, Chemicon Europe, $1: 400$ ), and two antibodies, mouse antirat cd11b (clone OX-42; CBL1512, Chemicon, Europe, $1: 400)$ and mouse antirat major histocompatibility complex (MHC) class II (clone OX6; ABD Serotec, Kidlington, UK) were used to identify microglia and activated microglia, respectively, since it has been reported that activated microglia express high levels of MHC class II [30]. After treatment with primary antibodies, sections were incubated with biotinylated 
secondary antibody (goat $\alpha$-rabbit or $\alpha$-mouse IgG, Vector Labs, Peterborough, UK) diluted $1: 200$ in PBS for 2 hours. Sections were incubated for a further 1 hour in horseradish peroxidase conjugate (Vectastain Elite ABC Kit, Vector Labs, Peterborough, UK), and antibody binding was visualised using a 3'3-diaminobenzidine (DAB) peroxidase staining kit with nickel enhancement (DAB peroxidase kit SK-4100, Vector Labs, Peterborough, UK) according to the manufacturer's instructions, followed by washing in distilled water. Each step was preceded by washing in PBS $(\mathrm{pH} 7.4,3 \times 5$ minutes $)$. Free-floating immunostained sections were mounted onto poly-L-lysine-coated slides (VWR, Lutterworth, UK) and allowed to adhere by airdrying. Mounted sections were rinsed with tap water before dehydration through a series of graded alcohol and xylene solutions, prior to application of coverslips using DPX mounting medium. Negative controls were performed in which the primary antibody was omitted and in which no immunostaining was observed, thereby confirming the specificity of the primary antibodies.

2.10. Cell Counting. The total number of TH-positive cells were counted manually rostro-caudally through the SNc in contiguous sections using a Nikon Eclipse E800 microscope (Nikon, Tokyo, Japan) connected to a JVC analogue camera and Image Pro Plus software (Media Cybernetics, Finchampstead, UK). The SNc was outlined using a manually traced region of interest $(\mathrm{ROI})$ at low magnification $(\times 4)$. The number of $\mathrm{TH}$ positive cells was counted in the contralateral and ipsilateral hemispheres at the level of third cranial nerve, within a $100 \mu \mathrm{m} \times 100 \mu \mathrm{m}$ counting area at high magnification $(\times 40)$ only within this defined ROI. Importantly, the level of the third cranial nerve provides a robust anatomical landmark where the SNc can be reliably delineated from the VTA, as previously described [31, 32]. Lesion size was then calculated as a percentage of the unlesioned contralateral hemisphere. Although not a stereological procedure, previous studies have shown that the 3rd nerve rootlets provide a reliable anatomical landmark at which the extent of cell loss is reflective of cell loss throughout the entire substantia nigra [32]. Further, manual cell counts assessed at the level of the third cranial nerve have been demonstrated to give equivalent results, not significantly different to that obtained from unbiased stereological estimates at the same level using an optical fractionator probe design [31]. These data strongly suggest that manual cell counting at the level of the third nerve is a viable method of determining cell loss and neuroprotection in this model [31].

To control for changes in $\mathrm{TH}$ expression in atrophic neurons, NeuN+ cell bodies in the SNc were counted in exactly the same manner as described above with reference to adjacent $\mathrm{TH}$-immunostained sections from the same animal. Two operators blinded to the treatment group under analysis performed all quantitative cell counting (H.C. and H.P.). At the same time as quantitative analysis was performed, representative photomicrographs were captured at $\times 40$ magnification using the same microscope and camera set-up and Image Pro Plus v.5.0 image analysis software (Media Cybernetics, Finchampstead, UK).
2.11. Measurement of Striatal Monoamines Using HPLC-ECD. Striatal monoamine content was analysed using HPLC-ECD, as previously described [19]. Briefly, the left and right striata were thawed, weighed and homogenised in $500 \mu \mathrm{l}$ of ice-cold homogenisation buffer $(50 \mathrm{mM}$ trichloroacetic acid, $0.5 \mathrm{mM}$ EDTA) containing $0.5 \mathrm{pmol} / \mathrm{mL} 3,4$-dihydroxybenzylamine hydrobromide (DHBA) as an internal standard. Striata were homogenised for 20 seconds by sonication (Soniprep, Sanyo, Loughborough, UK) and placed on ice for 10 minutes to allow complete extraction of monoamines, followed by centrifugation at $13000 \mathrm{~g}$ at $4^{\circ} \mathrm{C}$ for 10 minutes (Heraeus Centrifuges, Newport Pagnell, UK). Sample supernatant was filtered $(0.2 \mu \mathrm{m}$ PTFE filter, Whatman, Maidstone, UK) into HPLC vials (Chromacol, UK) and loaded onto an autosampler (Gina 50, Dionex, Camberley, UK) maintained at $5^{\circ} \mathrm{C}$. From each sample, $20 \mu \mathrm{L}$ were injected and analysed for dopamine (DA), dihydroxyphenylacetic acid (DOPAC), and homovanillic acid (HVA) content using a phosphate

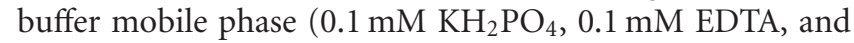
$1 \mathrm{mM}$ octyl sodium sulphonate, $10 \%$ methanol V/V, adjusted to $\mathrm{pH} 2.5$ with orthophosphoric acid) at a flow rate of $0.9 \mathrm{~mL} / \mathrm{min}$ on an Altex Ultrasphere $3 \mu \mathrm{m}$ ODS column $(4.6 \mathrm{~mm} \times 7.5 \mathrm{~cm}$, Beckman-Coulter, High Wycombe, UK). Samples were quantified by an electrochemical analytical cell (model 5011, ESA Analytical, Aylesbury, UK) attached to a Coulochem II electrochemical detector (ESA Analytical, Aylesbury, UK) with electrode one set at $-0.20 \mathrm{mV}$ and electrode two set at $+0.34 \mathrm{mV}$ with respect to the palladium reference electrode online with a dedicated PC-based data analysis programme (Chromeleon, Dionex, Camberley, UK). A set of standards for each monoamine and their metabolite were analysed after every fifth brain sample. Striata from both control and mGluR agonist-treated groups were analysed on the same day.

2.12. Statistical Analysis of Data. Data from vehicle and drugtreated groups were compared using two-tailed Student's $t$ test in Prism v5.0 software (GraphPad software, San Diego, CA, USA). All data are presented as mean \pm standard error of the mean (s.e.m) and differences were considered statistically significant at $P<.05$.

\section{Results}

3.1. Delayed Intranigral Injection of $2 R, 4 R-A P D C$ Provides Robust Neuroprotection against 6-OHDA Toxicity. The mean number of $\mathrm{TH}+$ cells in the unlesioned hemisphere of drug or vehicle-treated animals was comparable and not significantly different. Intranigral injection of 6-OHDA $(12 \mu \mathrm{g})$ resulted in a $\sim 70 \%$ reduction $(68.75 \pm 1.71[95 \%$ CI: $64.89 \%-74.62 \%$ ] of $\mathrm{TH}+$ cells in the ipsilateral SNc of vehicle-injected-animals $(n=8)$ relative to the contralateral hemisphere; Figure 1(a)). By contrast, in animals receiving intranigral injections of $2 R, 4 R$-APDC $(10 \mathrm{nmol})$ for 7 days, starting 48 hours post lesion, there was a significantly smaller reduction in $\mathrm{TH}+$ cells in the lesioned $\mathrm{SNc}$ compared to vehicle treated animals $(40.26 \pm 3.18 \%$ [95\% CI: $32.74 \%-$ $47.78 \%$ ] versus $68.75 \pm 1.71 \%$ [95\% CI: $64.89 \%-74.62 \%$ ]; 
$P<.001$; Figure 1(a)). To control for possible alterations in $\mathrm{TH}$ expression within atrophic neurons influencing the observed effects on $\mathrm{TH}+$ cell number in the $\mathrm{SNc}$, additional cell counts were performed in the same animals from each treatment group on tissue sections stained with the specific neuronal marker, NeuN. Critically, the loss of TH+ cells in the SNc was mirrored by an equivalent reduction in $\mathrm{NeuN}+$ cells in the lesioned SNc in vehicle-treated animals $(67.43 \pm 1.15 \%$ [95\% CI: $64.74 \%-69.95 \%])$. By contrast, a significantly smaller reduction in NeuN+ cells was observed in $2 R, 4 R$-APDC-treated animals compared to vehicle-treated animals $(37.89 \pm 4.03 \%$ [95\% CI: $28.36 \%-47.42 \%$ ] versus $(67.43 \pm 1.15 \%$ [95\% CI: 64.74\%-69.95\%]; $P<.001$; Figure 1(b)), consistent with the observed preservation of $\mathrm{TH}+$ cells in the lesioned SNc. These data are illustrated in representative photomicrographs of nigral $\mathrm{TH}+$ and $\mathrm{NeuN}+$ cells from both groups in Figures 1(c)-1(f).

No significant changes in monoamine content or turnover were observed in sham-lesioned animals (Table 1). Biochemical analysis of monoamine levels in the corpus striatum in each treatment group demonstrated that the neuroprotective effects of delayed $2 R, 4 R$-APDC treatment observed at the cellular level were associated with moderate, but statistically significant preservation of DA, DOPAC, and HVA concentrations in the ipsilateral corpus striatum compared to vehicle-injected controls $(P<.05$; Table 1$)$. Importantly, no significant differences were observed for any monoamine concentration in the contralateral hemisphere between vehicle and drug-treated animals $(P>.05$; Table 1$)$. A significant increase in striatal dopamine turnover ratio was observed in the lesioned striata of vehicle treated animals $(P<.05$; Table 2$)$, indicative of increased DA metabolism as an intrinsic compensatory response to the 6-OHDA lesion. Interestingly, in $2 R, 4 R$-APDC-injected animals, striatal DA turnover remained elevated in the lesioned hemisphere relative to the unlesioned side $(P<.05$; Table 2$)$, but importantly, this was significantly lower than that observed in the lesioned striatum of vehicle-treated animals $(P<.05$; Table 2).

3.2. Functional Recovery of Motor Behavior In Vivo Following Delayed Intranigral Injection of $2 R, 4 R-A P D C$. To functionally assess the neuroprotective effect of delayed intranigral $2 R, 4 R$-APDC injection $(10 \mathrm{nmol})$, spontaneous motor performance was assessed by observing forelimb use during exploratory rearing behaviour in the cylinder test. No significant forelimb use asymmetry was detected between treatment groups prior to 6-OHDA lesioning (data not shown). Both 2R,4R-APDC and vehicle-treated 6-OHDA lesioned animals displayed a significant shift towards use of the ipsilateral forelimb relative to the contralateral forelimb. However, this ipsilateral forelimb use bias was moderately, but significantly reduced in $2 R, 4 R$-APDC-injected animals compared to vehicle-treated animals $(42.6 \pm 4.1 \%$ [95\% CI: $32.79 \%-52.41 \%$ ] versus $66.4 \pm 5.9 \%$ [95\% CI: $52.78-80.07$ ]; $P<.05$; Figure 2(a)). In addition, the ipsilateral rotational responses to $(+)$-amphetamine were also investigated just prior to sacrifice. Injection of $5 \mathrm{mg} / \mathrm{kg}(+)$-amphetamine (i.p.) evoked a significant increase in net ipsilateral turns over the 60-minute recording session in vehicle-treated 6OHDA lesioned animals (Figure 3(b)), which was markedly attenuated in animals receiving delayed intranigral $2 R, 4 R-$ $\operatorname{APDC}(P<.001$; Figure $2(\mathrm{~b}))$.

3.3. Effect of Delayed Treatment with $2 R, 4 R-A P D C$ on Expression of Astroglial and Microglial Markers in the Substantia Nigra Pars Compacta. In vehicle-treated animals, dopaminergic neuronal death induced by 6-OHDA lesioning was accompanied by significant neuroinflammation. Indeed, compared to the intact unlesioned hemisphere (Figure 3(a)) a marked increase in the intensity of both GFAP and OX-42-immunostaining was observed in the lesioned SNc relative to the unlesioned hemisphere (Figure 3(b)). Both GFAP- and OX-42-positive (GFAP+/OX42+) cells in the unlesioned hemisphere displayed morphology typical of resting or quiescent cells (insets, Figures 3(a) and 3(e)). By contrast, in the lesioned hemisphere, a marked increased in GFAP+ and OX42+ cells displaying morphology typical of activated cells was observed, with astrocytes displaying thickened processes and a switch from ramified to ameboid morphology in microglia (insets, Figures 3(b) and 3(f)). Qualitative analysis suggests that delayed treatment with $2 R, 4 R$-APDC reduced the intensity of GFAP staining in the lesioned SNc, (Figures 3(c) and 3(d)) compared to vehicle-treated 6-OHDA lesioned animals (Figures 3(a) and $3(\mathrm{~b})$ ). In addition, fewer GFAP+ cells displayed activated morphology in the lesioned SNc of $2 R, 4 R$-APDC-treated animals compared to vehicle treated 6-OHDA lesioned animals (Figures 3(a)-3(d)). In addition, a clear decrease in the intensity of OX42+ staining in the lesioned SNc could be observed in $2 R, 4 R$-APDC injected animals (Figures $3(\mathrm{~g})$ and $3(\mathrm{~h})$ ), compared to vehicle-treated 6-OHDA lesioned animals (Figures 3(e) and 3(f)). Notably, qualitative analysis suggested a marked decrease in OX42+ cells displaying reactive morphology compared to vehicle-treated animals, although this was not completely abrogated (Figures 3(e) and $3(\mathrm{~h})$ ). Consistent with these observations, a clear increase in OX6 + cells displaying reactive morphology was also observed in the lesioned SNc of vehicle-treated 6-OHDA lesioned animals compared to the unlesioned hemisphere (Figures 4(a) and $4(\mathrm{~b})$ ), confirming the presence of activated microglia. Importantly, this was markedly, but not completely reduced in animals injected with $2 R, 4 R$-APDC (Figures $4(\mathrm{c}$ ) and $4(d))$.

\section{Discussion}

In the current study we have extended our initial preliminary observations [19] in vivo using the dual mGluR2/3 agonist $2 R, 4 R$-APDC in a rodent partial lesion 6-OHDA model of PD. Indeed, we now report that delayed (48 hour post lesion) subchronic intranigral administration of $2 R, 4 R$ APDC $(10 \mathrm{nmol})$ protects the rodent nigrostriatal system against 6-OHDA toxicity at the cellular (preservation of nigral $\mathrm{TH}+$ cell bodies) and biochemical level (preservation of striatal monoamine concentrations). This is effectively translated into functional recovery as demonstrated by 


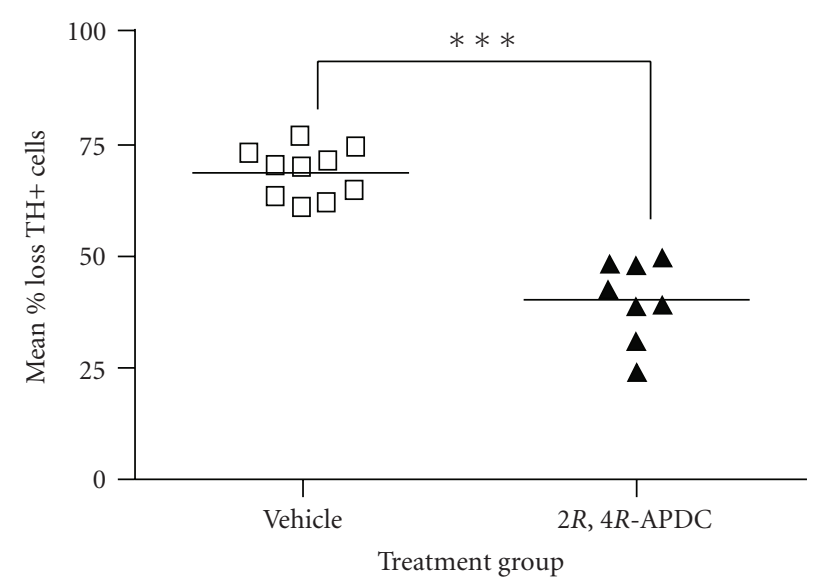

(a)

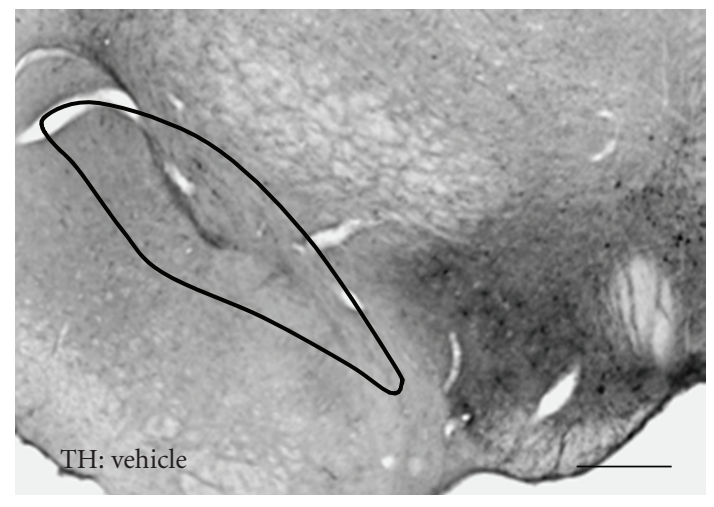

(c)

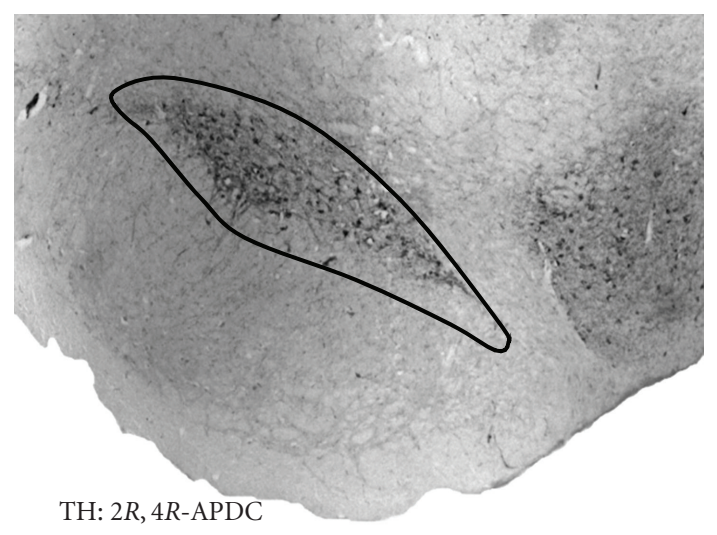

(e)

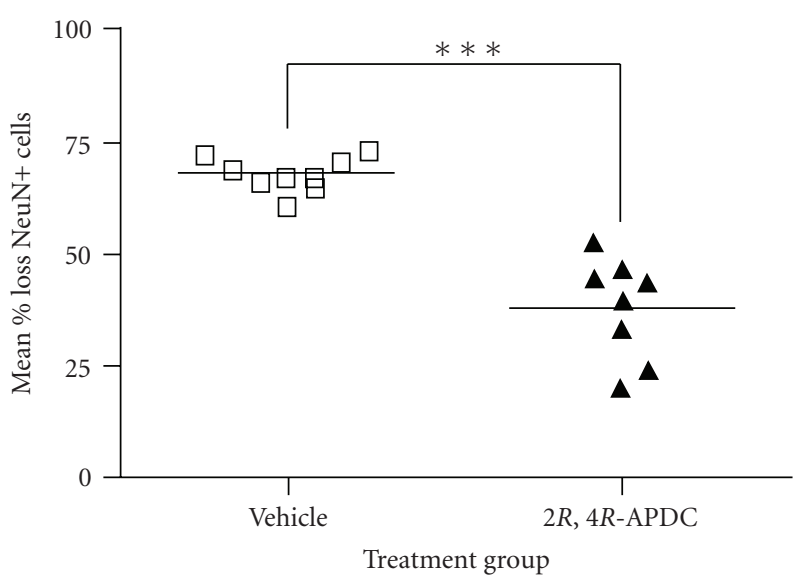

(b)

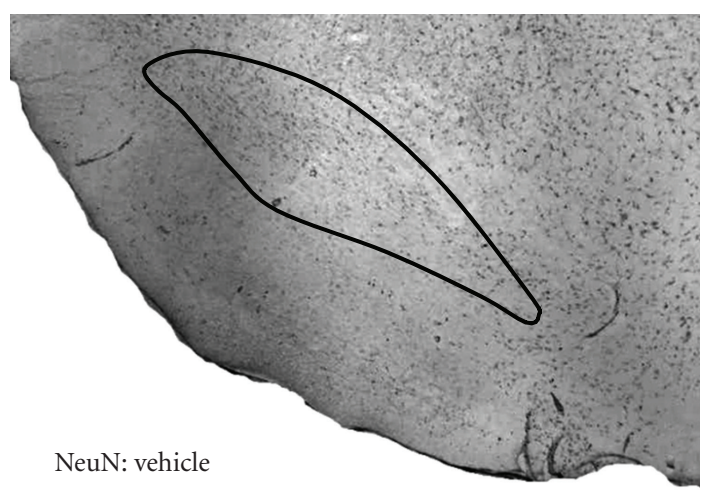

(d)

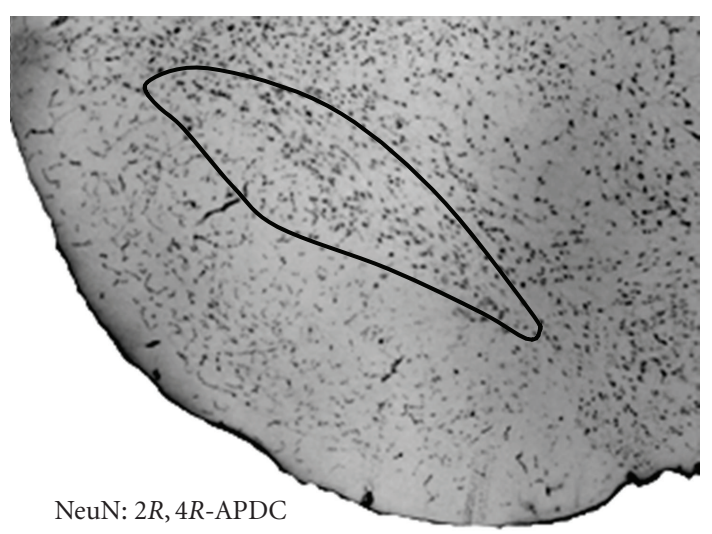

(f)

FIGURE 1: Delayed (48 hours post lesion) subchronic intranigral administration of $2 R, 4 R$-APDC (10 nmol in $4 \mu \mathrm{L})$ attenuates 6 -OHDA toxicity in vivo. Scatter plots showing (a) the mean percentage loss \pm s.e.m of nigral TH+ cells and (b) NeuN+cells in each treatment group (Vehicle $n=10,2 R, 4 R$-APDC, $n=8$ ). Note that 6-OHDA induces an equivalent reduction of both TH and NeuN+cells in the SNc, which is significantly attenuated following delayed administration of $2 R, 4 R$-APDC $(10 \mathrm{nmol})$. ${ }^{* * *} P<.001$, vehicle versus $2 R, 4 R$-APDC $(2$-tailed students $t$-test). (c)-(f) Representative photomicrographs $(\times 40)$ of DAB/peroxidase staining for $\mathrm{TH}$ and NeuN+cells in the SNc from each treatment group, also showing the ROI encompassing the SNc used for cell counting, scale bar $200 \mu \mathrm{m}$. (c) TH, 6-OHDA + vehicle, (d) NeuN,6-OHDA + vehicle, (e) TH, 6-OHDA + 2R,4R-APDC, (f) NeuN, 6-OHDA + 2R,4R-APDC.

improved forelimb asymmetry scores and correction of amphetamine-induced rotational asymmetry. In addition, these effects were associated with a qualitative decrease in microglial activation in the SNc. Importantly, in animals receiving $2 R, 4 R$-APDC post lesion, $\mathrm{NeuN}+$ and $\mathrm{TH}+$ cell loss was attenuated in parallel in the ipsilateral SNc, strongly supporting a neuroprotective effect, rather than alterations in $\mathrm{TH}$ expression in atrophic neurones. However, although 
TABLE 1: Mean striatal concentrations of dopamine (DA), dihydroxyphenylacetic acid (DOPAC), and homovanillic acid (HVA) as measured by HPLC-ECD in the unlesioned and lesioned striata in each treatment group. Data shown are mean ng/mg wet weight tissue \pm s.e.m. ${ }^{*} P<.05,{ }^{* *} P<.01$ unlesioned versus lesioned hemisphere, ${ }^{\dagger} P<.05,{ }^{\dagger \dagger} P<.012 R, 4 R$-APDC versus vehicle-treated animals [10]. Drug concentration in nmol (final injection volume $4 \mu \mathrm{L}$ ).

\begin{tabular}{lcccccc}
\hline $\begin{array}{l}\text { Treatment } \\
\text { group }\end{array}$ & $\begin{array}{c}\text { [DA] Unlesioned } \\
\mathrm{ng} / \mathrm{mL}\end{array}$ & $\begin{array}{c}\text { [DA] Lesioned } \\
\mathrm{ng} / \mathrm{mL}\end{array}$ & $\begin{array}{c}\text { [DOPAC] Unlesioned } \\
\mathrm{ng} / \mathrm{mL}\end{array}$ & $\begin{array}{c}\text { [DOPAC] Lesioned } \\
\mathrm{ng} / \mathrm{mL}\end{array}$ & $\begin{array}{c}\text { [HVA] Unlesioned } \\
\mathrm{ng} / \mathrm{mL}\end{array}$ & $\begin{array}{c}\text { [HVA] Lesioned } \\
\mathrm{ng} / \mathrm{mL}\end{array}$ \\
\hline Vehicle & $7.26 \pm 0.16$ & $1.042 \pm 0.23^{* *}$ & $1.99 \pm 0.13$ & $0.59 \pm 0.15^{* *}$ & $0.74 \pm 0.07$ & $0.33 \pm 0.09^{* *}$ \\
$2 R, 4 R$-APDC & $7.87 \pm 0.18$ & $3.40 \pm 0.54^{* *+\dagger}$ & $1.46 \pm 0.10$ & $0.81 \pm 0.07^{* * \dagger}$ & $0.65 \pm 0.04$ & $0.45 \pm 0.06^{* * \dagger}$ \\
\hline
\end{tabular}

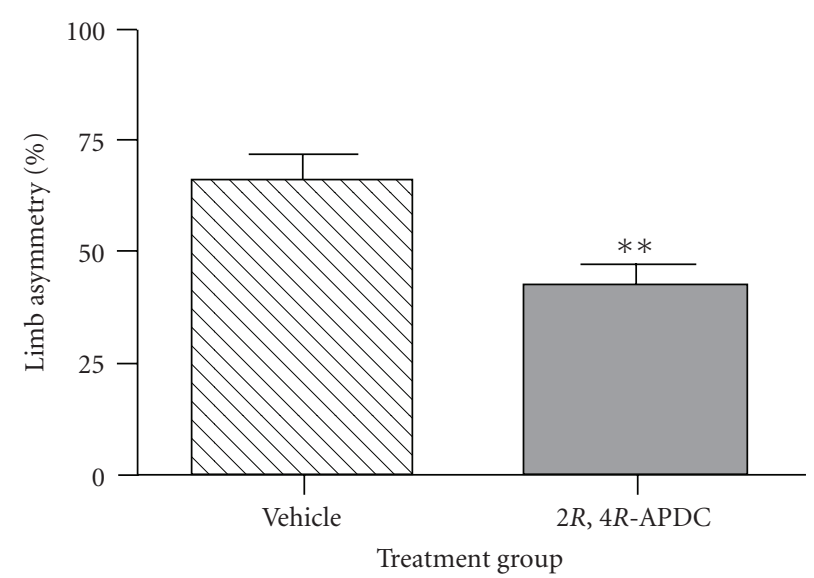

(a)

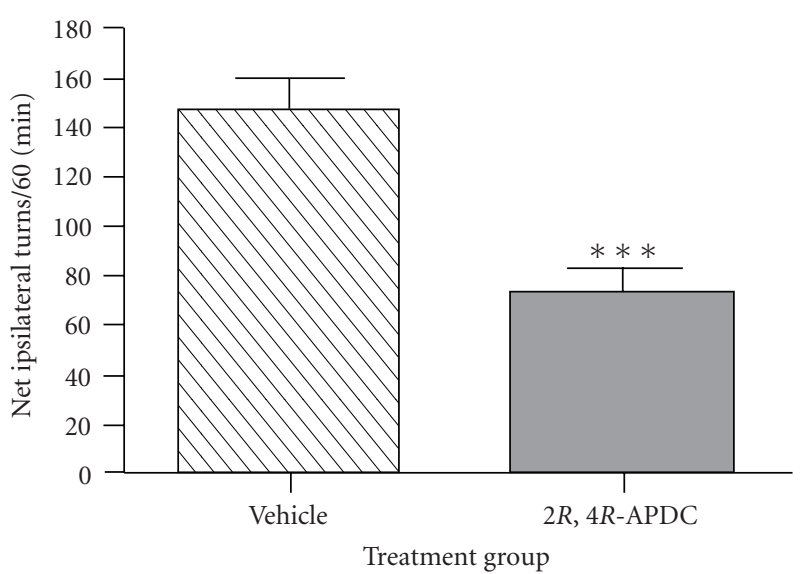

(b)

FIGURE 2: Neuroprotection against 6-OHDA toxicity following delayed subchronic intranigral administration of 2R,4R-APDC (10 nmol in $4 \mu \mathrm{L}$ ) translates into functional recovery of motor deficits. (a) Bar graph of mean percentage forelimb use asymmetry \pm s.e.m in vehicle and $2 R, 4 R$-APDC $(10 \mathrm{nmol})$-treated animals, ${ }^{* *} P<0.01$, vehicle versus $2 R, 4 R$-APDC. (b) Bar graph of amphetamine-induced rotational behavior, expressed as net ipsilateral turns over 60 minutes \pm s.e.m in vehicle and $2 R, 4 R$-APDC-treated animals, ${ }^{* * *} P<.001$, vehicle versus $2 R, 4 R$-APDC.

TABle 2: Measurement of striatal dopamine metabolism in the unlesioned and lesioned striata in each treatment group. Data are expressed as mean dopamine turnover ratio \pm s.e.m calculated by the expression (DOPAC + HVA/DA) using mean pmol/mg wet weight tissue values for DA, DOPAC, and HVA, respectively. ${ }^{*} P<$ .05 , unlesioned versus lesioned hemisphere. ${ }^{\dagger} P<.05,2 R, 4 R$-APDC versus vehicle-treated animal [10]. Drug concentrations in nmol (final injection volume $4 \mu \mathrm{L}$ ).

\begin{tabular}{lcc}
\hline $\begin{array}{l}\text { Treatment } \\
\text { group }\end{array}$ & $\begin{array}{c}\text { DA turnover ratio } \\
\text { Unlesioned }\end{array}$ & $\begin{array}{c}\text { DA turnover ratio } \\
\text { lesioned }\end{array}$ \\
\hline Vehicle & $0.30 \pm 0.04$ & $0.45 \pm 0.12^{*}$ \\
$2 R, 4 R$-APDC & $0.26 \pm 0.10$ & $0.31 \pm 0.04^{* \dagger}$ \\
\hline
\end{tabular}

intranigral treatment with $2 R, 4 R$-APDC lowered the intrinsic compensatory increases in striatal DA metabolism compared to vehicle-treated animals, this was not completely reversed. Thus, we cannot exclude the possibility that the neuroprotective effects of $2 R, 4 R$-APDC on striatal DA and its metabolites is not partly due to intrinsic compensatory mechanisms. Furthermore, it will be important in future studies to determine whether these data are replicated upon systemic administration and in a more progressive model of $\mathrm{PD}$, such as the intrastriatal 6-OHDA model [33]. Notably,
$2 R, 4 R$-APDC did not completely prevent 6-OHDA toxicity in vivo, consistent with previous in vivo studies [14, 17-19].

Preliminary data from our laboratory have shown the neuroprotective effects of $2 R, 4 R$-APDC are abrogated by coadministration with EGLU, a highly selective group II mGluR antagonist [34]. These observations strongly suggesting the beneficial effects of $2 R, 4 R$-APDC effects are mediated through selective activation of mGluR2/3 in vivo (Vernon et al., unpublished observations). Interestingly, the relatively weak neuroprotective effects observed in previous studies [14, 17-19] may be explained by evidence suggesting that $\mathrm{mGlu} 2$ and 3 receptors have divergent functions in vivo [15]. Indeed, in an elegant knock-out mice study, Corti and colleagues (2007) demonstrated that the systemic administration of the dual mGluR2/3 agonist LY379268 protected striatal neurons against NMDA toxicity in wildtype and mGluR2 $2^{-/-}$mice but not in mGluR3 ${ }^{-/-}$mice [15]. Moreover, LY379268 was neuroprotective against nigrostriatal degeneration induced by low doses of 1-methyl-4phenyl-1,2,3,6-tetrahydropyridine (MPTP) in mGluR2 ${ }^{-/-}$ mice, strongly suggesting that these in vivo neuroprotective effects are dependent on the activation of mGluR3 [15]. Since LY379268 was only neuroprotective in $\mathrm{mGlu} 2^{-/-}$mice, these data suggest that activation of mGluR2 in wild-type mice may counterbalance the protective activity of the drug [15]. This hypothesis is supported by detailed in vitro 


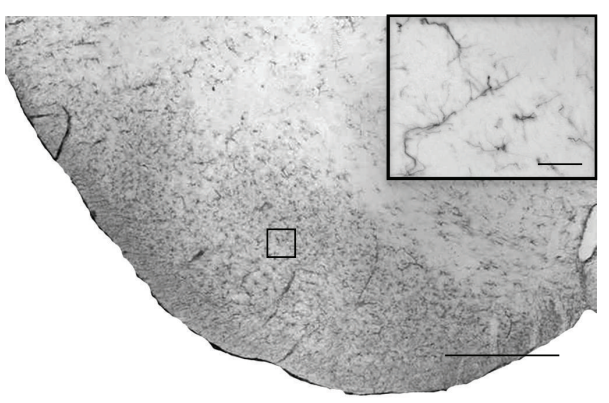

(a)

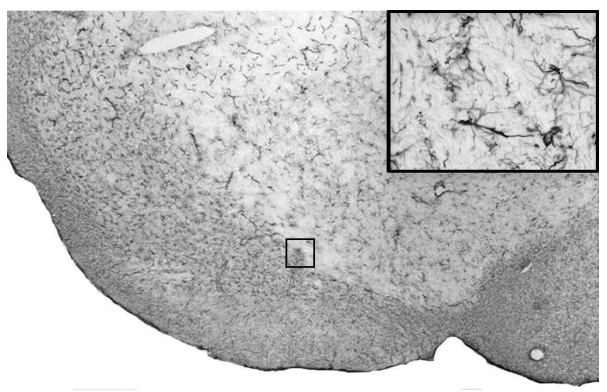

(c)

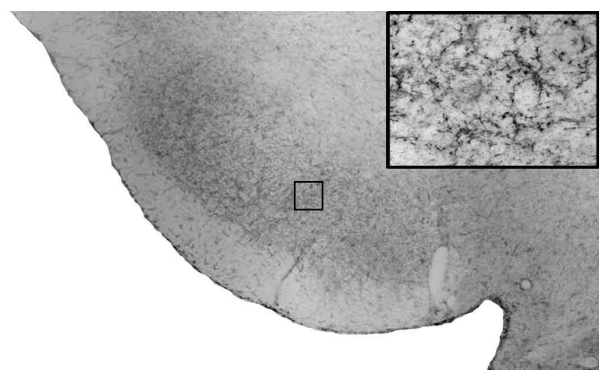

(e)

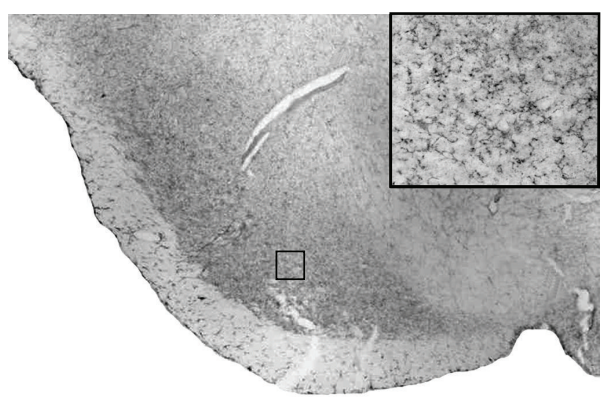

(g)

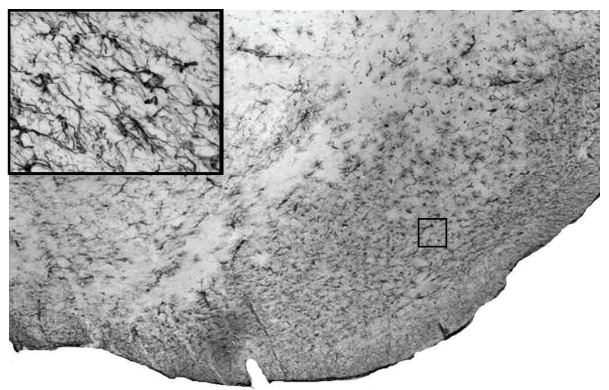

(b)

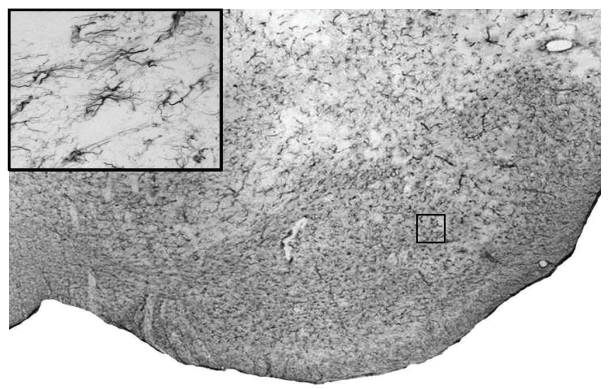

(d)

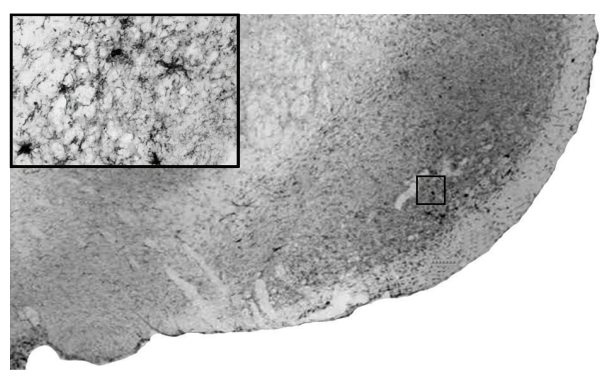

(f)

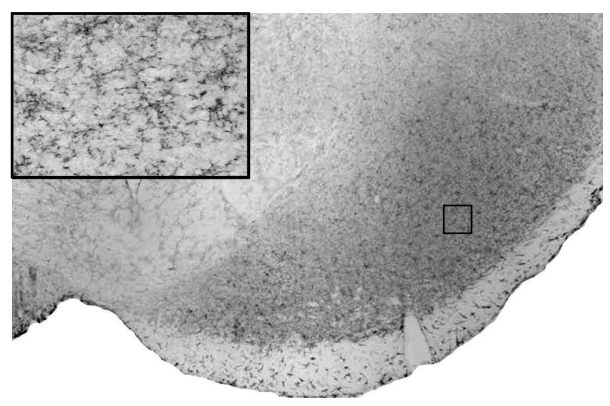

(h)

FIGURE 3: Neuroprotection against 6-OHDA toxicity following delayed subchronic intranigral administration of $2 R, 4 R$-APDC is associated with qualitative reductions in inflammatory markers. Representative photomicrographs $(\times 40)$ of DAB/peroxidase staining for GFAP (astrocytosis) and OX-42 (microgliosis) in the SNc from each treatment group (scale bar $200 \mu \mathrm{m}$.). Panels (a) $-(\mathrm{g})$ show the intensity and distribution of GFAP+ and OX-42+ cells in the contralateral control SN of vehicle (a), (c) and (e), (g) 2R,4R-APDC (10 nmol)-injected animals. Panels (b)-(h) show the intensity and distribution of GFAP+ and OX-42+ cells in the Lesioned SNc of vehicle (b), (d) and (e), (g) $2 R, 4 R$-APDC (10 nmol)-injected animals. (Insets (a)-(h)), $\times 60$, enlargements of outlined areas (scale bar $10 \mu \mathrm{m})$ ). Note the apparent reduction in intensity of GFAP+ and OX-42+ staining in $2 R, 4 R$-APDC-injected animals compared to vehicle-treated animals. Further, $2 R, 4 R$-APDC treatment appeared to reduce the number of cells with morphology characteristic for reactive astrocytes or activated microglia in the SNc in comparison to vehicle-treated controls. 


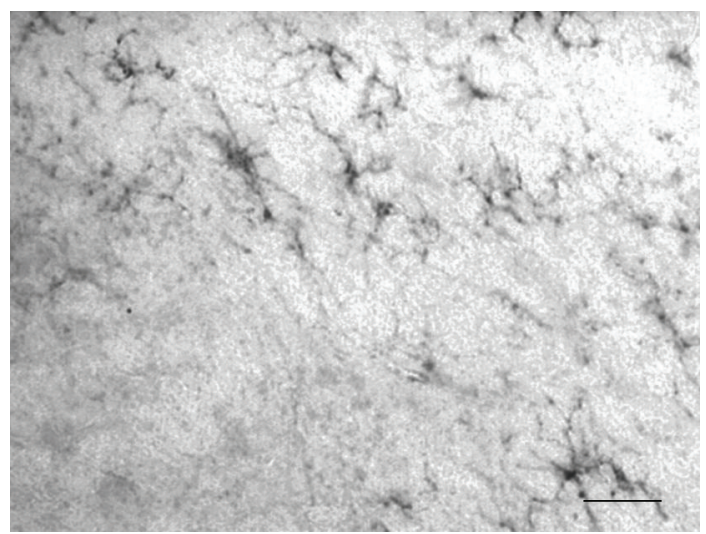

(a)

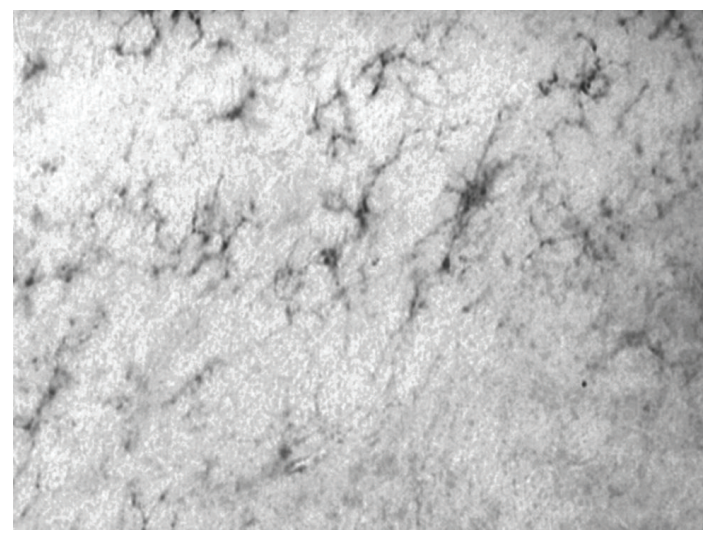

(c)

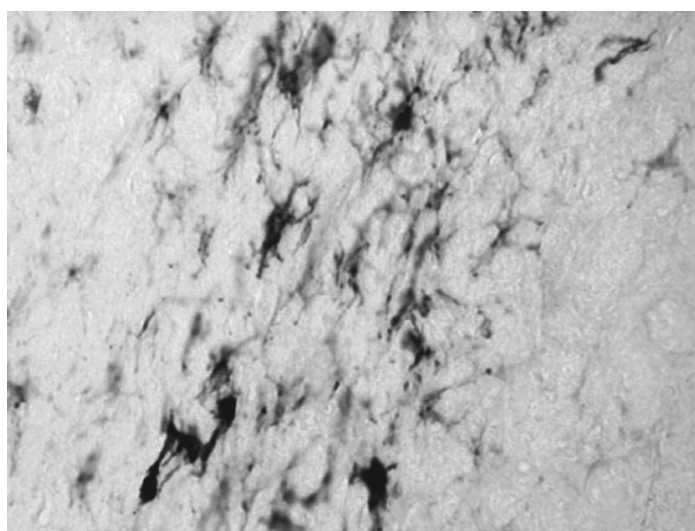

(b)

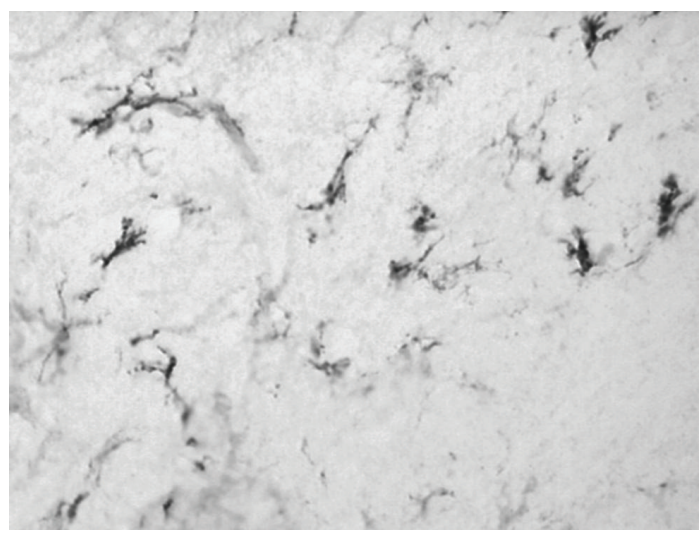

(d)

FIGURE 4: Neuroprotection against 6-OHDA toxicity following delayed subchronic intranigral administration of $2 R, 4 R$-APDC is associated with reduced numbers of activated microglia in the SNc. Representative photomicrographs $(\times 40)$ of DAB/peroxidase staining for OX-6, a marker of activated microglia in the SNc from each treatment group (scale bar $200 \mu \mathrm{m}$.). (a) 6-OHDA + vehicle, unlesioned SNc, (b) 6-OHDA + vehicle, lesioned SNc, (c) 6-OHDA + 2R,4R-APDC (10 nmol) unlesioned SNc, (d) 6-OHDA + 2R,4R-APDC (10 nmol) lesioned SNc. Note the increase in intensity of OX-6 staining and the increase in OX-6+ cells displaying morphology of activated microglia in the lesioned SNc of vehicle-treated animal, which appears markedly reduced in animals treated with $2 R, 4 R$-APDC.

experiments in the same study, which provided evidence that activation of mGluR2 may enhance NMDA toxicity in mixed cortical cultures [15]. Thus, in vitro and in vivo evidence converges to suggest that mGluR3 mediates the neuroprotective activity of $\mathrm{mGluR} 2 / 3$ receptor agonists in vivo and that a combined activation of mGluR2 and mGluR3 receptors may therefore limit the extent of neuroprotection observed [15]. Conversely, it is interesting to note that mGluR2 activation alone is required for novel antipsychotic actions of dual $\mathrm{mGluR} 2 / 3$ agonists in vivo $[35,36]$. However, it is clear that dual mGluR2/3 agonists such as LY367385 and indeed, $2 R, 4 R$-APDC might be predicted to have less neuroprotective efficacy than mGluR3 agonists alone [15].

This also has implications for the potential antiparkinsonian effects of these compounds in preclinical models of PD. In the current study, we provide preliminary evidence that neuroprotection following delayed, subchronic intranigral administration of $2 R, 4 R$-APDC translated into functional recovery, confirming a neuroprotective effect at the behavioural level. These data are somewhat consistent with previous data in which systemic administration of the dual
mGluR2/3 agonist LY379268 weakly ameliorated reserpineinduced akinesia, but did not reverse amphetamine rotational asymmetry in 6-OHDA lesioned rats, despite modest neuroprotective effects [18]. Interestingly, intrastriatal injection of $2 R, 4 R$-APDC did not reverse haloperidolinduced catalepsy or muscle rigidity in rats [21]. These divergent results may be explained by data demonstrating that dual mGluR2/3 agonists, (including LY379268 and $2 R, 4 R$-APDC) inhibit not only glutamate, but also DA release in the striatum in vivo [37]. Interestingly, LY379268 also displays partial agonist activity at $\mathrm{DA} \mathrm{D}_{2}$ receptors in vitro and in vivo $[38,39]$. Thus, following systemic or intrastriatal administration, dual mGluR2/3 agonists may bind to presynaptic $\mathrm{DA} \mathrm{D}_{2}$ receptors thereby decreasing DA release in the striatum and inhibiting locomtion $[38,39]$. Whether 2R,4R-APDC has partial agonist activity at DA $\mathrm{D}_{2}$ receptors is unknown. However, recent data suggest that Group II mGluR, in particular mGluR2, are closely associated with $\mathrm{DA} \mathrm{D}_{2}$ receptors and may regulate their number and activity in the striatum $[40,41]$. Indeed, the number of $\mathrm{D}_{2}$ receptors in the striatum is markedly elevated 
in mGluR2 and mGluR3 knock-out mice [40, 41]. Moreover, in vitro striatal homogenates from mGluR2 knock-out mice displayed significantly greater supersensitivity responses to the $\mathrm{D}_{2}$ receptor agonist $(+)$ PHNO when compared to those from mGluR3 knock-outs, suggesting that mGluR2 and DA $\mathrm{D}_{2}$ receptors are functionally linked in vivo [40].

Taken together, these data suggest that translation of neuroprotection into functional recovery following dual mGluR2/3 agonist treatment is critically dependent on the route of drug administration. Indeed, our own data demonstrate that this may be observed following activation of nigral mGluR2/3 in isolation. By contrast, following systemic administration, confounding actions in other brain regions particularly, but not exclusively, in the striatum might occlude this effect [18]. Notably again, this appears predominantly due to the activation of mGluR2 [38]. Thus, these data reinforce the prediction that mGluR3 selective compounds may be more efficacious as both neuroprotective and antiparkinsonian agents, although this remains to be tested in vivo.

In the current study we also observed that the neuroprotective effects of $2 R, 4 R$-APDC were associated with a qualitative decrease in microglial markers in the SNc. Interestingly, in vitro, activation of mGluR3 expressed in microglia inhibits microglial activation and secretion of neurotoxic cytokines [42, 43]. Conversely, activation of microglial mGluR2 enhances microglial toxicity in vitro [42, 43]. Again, these data are consistent with divergent roles of mGluR2 and 3 in vivo [15] and may certainly also contribute to the modest neuroprotective effects of $2 R, 4 R$-APDC in vivo by counterbalancing beneficial effects of mGluR3 activation. Nevertheless, based on this observation it is tempting to speculate that the neuroprotective effects of $2 R, 4 R$-APDC and other dual mGluR2/3 agonists in vivo may involve antiinflammatory mechanisms, including prevention of toxic microglial activation, alongside documented effects such as promoting secretion of trophic-factors from astrocytes [1215]. However, these data should be interpreted cautiously, as decreases in microglial markers may simply reflect a secondary effects due to neuroprotection by $2 R, 4 R$-APDC through alternative mechanisms. Nevertheless, these initial observations suggest that further studies on the effects of mGluR2/3 agonists on microglial response in vivo may be warranted.

\section{Conclusions}

In conclusion, this study extends our preliminary findings [19] and provides evidence that subchronic intranigral injection of the dual mGluR2/3 agonist $2 R, 4 R$-APDC robustly attenuates dopaminergic neurodegeneration in vivo, even when treatment initiation is delayed. Furthermore, this translates into modest functional recovery, at least following intranigral administration in vivo. Moreover, the neuroprotective effects of $2 R, 4 R$-APDC may involve modification of the inflammatory response as evidenced by a qualitative reduction on the expression of microglial markers in the $\mathrm{SNc}$. However, we fully acknowledge that the significance of these data should be interpreted with the caveat that we have utilised a dual mGluR2/3 agonists and thus any beneficial effects are likely to be influenced by confounding effects due to activation of mGluR2 in vivo [15]. Nevertheless, these data provide further evidence for the rationale of targeting Group II mGluR in PD, although it will be vitally important in future studies to repeat these experiments using mGluR3-selective compounds as these become available. Overall, this also has implications for the clinical use of dual mGluR2/3 agonists for the treatment of neurodegenerative diseases. Indeed, on the basis of currently available data from pre-clinical studies, orthosteric nonselective mGluR2/3 agonists may be of limited clinical use as neuroprotective/antiparkinsonian agents due to confounding effects at mGluR2 in vivo [15]. The development of a novel class of compounds, known as positive allosteric modulators (PAMs), which do not activate the receptor directly, but bind to a site distinct from the glutamate-binding site, with the effect of increasing the response of the receptor to endogenous glutamatergic tone, may offer a solution to this problem, since such binding sites are often less constrained and more amenable to discovery of novel, subtype-selective compounds, compared to targeting the glutamate binding site [44]. Indeed, a number of PAMs selective for mGluR2 have been developed, which may offer an exciting alternative approach to dual mGluR2/3 agonists at least for their antipsychotic or anxiolytic indications [44-47]. Notably however, this has not been matched by a similar development of mGluR3 selective PAMs. Thus the development of mGluR3 selective compounds is eagerly awaited and could represent a crucial step towards the potential use of these compounds for use in neurodegenerative conditions such as PD.

\section{Nonstandard Abbreviations}

\section{R,4R-APDC: $2 R, 4 R$-4-aminopyrrolidine-2,4- dicarboxylate \\ EGLU: $\quad(S)$ - $\alpha$-Ethylglutamic acid \\ DCG-IV: $\quad\left(2 S, 2^{\prime} R, 3^{\prime} R\right)-2-\left(2^{\prime}, 3^{\prime}-\right.$ Dicarboxycyclopropyl)glycine}

HPLC-ECD: high performance liquid chromatographyelectrochemical detection

LY379268: $\quad(1 R, 4 R, 5 S, 6 R)-4$-Amino-2oxabicyclo[3.1.0] hexane-4,6-dicarboxylic acid

LY354740: $\quad(1 S, 2 S, 5 R, 6 S)-2-$ aminobicyclo[3.1.0]hexane-2,6-dicarboxylic acid

6-OHDA: 6-hydroxydopamine; mGluR, metabotropic glutamate receptor

MTN: $\quad$ medial terminal nucleus

NMDAR: $\quad N$-methyl- $D$-aspartate receptor

PD: $\quad$ Parkinson's disease

+PHNO: 4-propyl-9-hydroxynaphthoxazine

SNc: $\quad$ substantia nigra pars compacta

SNr: $\quad$ substantia nigra pars reticulata

STN: $\quad$ subthalamic nucleus

VTA: ventral tegmental area. 


\section{Acknowledgments}

This work was funded jointly by grants from the Medical Research Council and Dorothy Hodgkin Trust, whom the authors would like to thank for their generous financial assistance.

\section{References}

[1] J. E. Ahlskog, "I can't get no satisfaction: still no neuroprotection for Parkinson disease," Neurology, vol. 69, no. 15, pp. 1476-1477, 2007.

[2] A. H. V. Schapira, E. Bezard, J. Brotchie, et al., "Novel pharmacological targets for the treatment of Parkinson's disease," Nature Reviews Drug Discovery, vol. 5, no. 10, pp. 845-854, 2006.

[3] P. Jenner and C. W. Olanow, "The pathogenesis of cell death in Parkinson's disease," Neurology, vol. 66, no. 10, supplement 4, pp. S24-S36, 2006.

[4] P. J. Conn, G. Battaglia, M. J. Marino, and F. Nicoletti, "Metabotropic glutamate receptors in the basal ganglia motor circuit," Nature Reviews Neuroscience, vol. 6, no. 10, pp. 787798, 2005.

[5] P. J. Conn, C. W. Lindsley, and C. K. Jones, "Activation of metabotropic glutamate receptors as a novel approach for the treatment of schizophrenia," Trends in Pharmacological Sciences, vol. 30, no. 1, pp. 25-31, 2009.

[6] F. Nicoletti, V. Bruno, A. Copani, G. Casabona, and T. Knöpfel, "Metabotropic glutamate receptors: a new target for the therapy of neurodegenerative disorders?" Trends in Neurosciences, vol. 19, no. 7, pp. 267-271, 1996.

[7] S. T. Patil, L. Zhang, F. Martenyi, et al., "Activation of mGlu2/3 receptors as a new approach to treat schizophrenia: a randomized Phase 2 clinical trial," Nature Medicine, vol. 13, no. 9, pp. 1102-1107, 2007.

[8] E. Dunayevich, J. Erickson, L. Levine, R. Landbloom, D. D. Schoepp, and G. D. Tollefson, "Efficacy and tolerability of an mGlu2/3 agonist in the treatment of generalized anxiety disorder," Neuropsychopharmacology, vol. 33, no. 7, pp. 16031610, 2008.

[9] S. R. Bradley, M. J. Marino, M. Wittmann, et al., "Activation of group II metabotropic glutamate receptors inhibits synaptic excitation of the substantia nigra pars reticulata," Journal of Neuroscience, vol. 20, no. 9, pp. 3085-3094, 2000.

[10] L. Wang, S. T. Kitai, and Z. Xiang, "Modulation of excitatory synaptic transmission by endogenous glutamate acting on presynaptic group II mGluRs in rat substantia nigra compacta," Journal of Neuroscience Research, vol. 82, no. 6, pp. 778787, 2005.

[11] M. Wittmann, M. J. Marino, and P. J. Conn, "Dopamine modulates the function of group II and group III metabotropic glutamate receptors in the substantia nigra pars reticulata," Journal of Pharmacology and Experimental Therapeutics, vol. 302, no. 2, pp. 433-441, 2002.

[12] V. Bruno, G. Battaglia, G. Casabona, A. Copani, F. Caciagli, and F. Nicoletti, "Neuroprotection by glial metabotropic glutamate receptors is mediated by transforming growth factor- $\beta$," Journal of Neuroscience, vol. 18, no. 23, pp. 9594 9600, 1998.

[13] M. D’Onofrio, L. Cuomo, G. Battaglia, et al., "Neuroprotection mediated by glial group-II metabotropic glutamate receptors requires the activation of the MAP kinase and the phosphatidylinositol-3-kinase pathways," Journal of Neurochemistry, vol. 78, no. 3, pp. 435-445, 2001.

[14] G. Battaglia, G. Molinaro, B. Riozzi, et al., "Activation of mGlu3 receptors stimulates the production of GDNF in striatal neurons," PLoS One, vol. 4, no. 8, article e6591, 2009.

[15] C. Corti, G. Battaglia, G. Molinaro, et al., "The use of knock-out mice unravels distinct roles for mGlu2 and mGlu3 metabotropic glutamate receptors in mechanisms of neurodegeneration/neuroprotection," Journal of Neuroscience, vol. 27, no. 31, pp. 8297-8308, 2007.

[16] E. R. Matarredona, M. Santiago, J. L. Venero, J. Cano, and A. Machado, "Group II metabotropic glutamate receptor activation protects striatal dopaminergic nerve terminals against $\mathrm{MPP}^{+}$-induced neurotoxicity along with brain-derived neurotrophic factor induction," Journal of Neurochemistry, vol. 76, no. 2, pp. 351-360, 2001.

[17] G. Battaglia, C. L. Busceti, F. Pontarelli, et al., "Protective role of group-II metabotropic glutamate receptors against nigrostriatal degeneration induced by 1-methyl-4-phenyl-1,2,3,6tetrahydropyridine in mice," Neuropharmacology, vol. 45, no. 2, pp. 155-166, 2003.

[18] T. K. Murray, M. J. Messenger, M. A. Ward, et al., "Evaluation of the mGluR2/3 agonist LY379268 in rodent models of Parkinson's disease," Pharmacology Biochemistry and Behavior, vol. 73, no. 2, pp. 455-466, 2002.

[19] A. C. Vernon, S. Palmer, K. P. Datla, V. Zbarsky, M. J. Croucher, and D. T. Dexter, "Neuroprotective effects of metabotropic glutamate receptor ligands in a 6-hydroxydopamine rodent model of Parkinson's disease," European Journal of Neuroscience, vol. 22, no. 7, pp. 1799-1806, 2005.

[20] J. Konieczny, K. Ossowska, S. Wolfarth, and A. Pilc, "LY354740, a group II metabotropic glutamate receptor agonist with potential antiparkinsonian properties in rats," Naunyn-Schmiedeberg's Archives of Pharmacology, vol. 358, no. 4, pp. 500-502, 1998.

[21] K. Ossowska, J. Konieczny, J. Wardas, et al., "An influence of ligands of metabotropic glutamate receptor subtypes on parkinsonian-like symptoms and the striatopallidal pathway in rats," Amino Acids, vol. 32, no. 2, pp. 179-188, 2007.

[22] L. Dawson, A. Chadha, M. Megalou, and S. Duty, "The group II metabotropic glutamate receptor agonist, DCG-IV, alleviates akinesia following intranigral or intraventricular administration in the reserpine-treated rat," British Journal of Pharmacology, vol. 129, no. 3, pp. 541-546, 2000.

[23] D. D. Schoepp, D. E. Jane, and J. A. Monn, "Pharmacological agents acting at subtypes of metabotropic glutamate receptors," Neuropharmacology, vol. 38, no. 10, pp. 1431-1476, 1999.

[24] P. A. Watson, The Rat Brain in Stereotaxic Co-ordinates, Academic Press, New York, NY, USA, 2007.

[25] A. C. Vernon, V. Zbarsky, K. P. Datla, D. T. Dexter, and M. J. Croucher, "Selective activation of group III metabotropic glutamate receptors by L-(+)-2-amino-4-phosphonobutryic acid protects the nigrostriatal system against 6-hydroxydopamine toxicity in vivo," Journal of Pharmacology and Experimental Therapeutics, vol. 320, no. 1, pp. 397-409, 2007.

[26] D. D. Schoepp, C. R. Salhoff, R. A. Wright, et al., "The novel metabotropic glutamate receptor agonist 2R,4R-APDC potentiates stimulation of phosphoinositide hydrolysis in the rat hippocampus by 3,5-dihydroxyphenylglycine: Evidence for a synergistic interaction between group 1 and group 2 receptors," Neuropharmacology, vol. 35, no. 12, pp. 1661-1672, 1996. 
[27] J. R. Cannon, R. F. Keep, T. Schallert, Y. Hua, R. J. Richardson, and G. Xi, "Protease-activated receptor-1 mediates protection elicited by thrombin preconditioning in a rat 6-hydroxydopamine model of Parkinson's disease," Brain Research, vol. 1116, no. 1, pp. 177-186, 2006.

[28] J. L. Tillerson, A. D. Cohen, J. Philhower, G. W. Miller, M. J. Zigmond, and T. Schallert, "Forced limb-use effects on the behavioral and neurochemical effects of 6-hydroxydopamine," Journal of Neuroscience, vol. 21, no. 12, pp. 4427-4435, 2001.

[29] U. Ungerstedt and G. W. Arbuthnott, "Quantitative recording of rotational behavior in rats after 6-hydroxy-dopamine lesions of the nigrostriatal dopamine system," Brain Research, vol. 24, no. 3, pp. 485-493, 1970.

[30] W. J. Streit, M. B. Graeber, and G. W. Kreutzberg, "Functional plasticity of microglia: a review," Glia, vol. 1, no. 5, pp. 301307, 1988.

[31] S. Bukhatwa, M. M. Iravani, B.-Y. Zeng, J. D. Cooper, S. Rose, and P. Jenner, "An immunohistochemical and stereological analysis of PSI-induced nigral neuronal degeneration in the rat," Journal of Neurochemistry, vol. 109, no. 1, pp. 52-59, 2009.

[32] M. M Iravani, K. Kashefi, P. Mander, S. Rose, and P. Jenner, "Involvement of inducible nitric oxide synthase in inflammation-induced dopaminergic neurodegeneration," Neuroscience, vol. 110, no. 1, pp. 49-58, 2002.

[33] H. Sauer and W. H. Oertel, "Progressive degeneration of nigrostriatal dopamine neurons following intrastriatal terminal lesions with 6-hydroxydopamine: a combined retrograde tracing and immunocytochemical study in the rat," Neuroscience, vol. 59, no. 2, pp. 401-415, 1994.

[34] D. E. Jane, N. K. Thomas, H.-W. Tse, and J. C. Watkins, "Potent antagonists at the L-AP4- and (1S,3S)-ACPD-sensitive presynaptic metabotropic glutamate receptors in the neonatal rat spinal cord," Neuropharmacology, vol. 35, no. 8, pp. 1029 1035, 1996.

[35] C. J. Swanson, M. Bures, M. P. Johnson, A.-M. Linden, J. A. Monn, and D. D. Schoepp, "Metabotropic glutamate receptors as novel targets for anxiety and stress disorders," Nature Reviews Drug Discovery, vol. 4, no. 2, pp. 131-144, 2005.

[36] M. L. Woolley, D. J. Pemberton, S. Bate, C. Corti, and D. N. C. Jones, "The mGlu2 but not the mGlu3 receptor mediates the actions of the mGluR2/3 agonist, LY379268, in mouse models predictive of antipsychotic activity," Psychopharmacology, vol. 196, no. 3, pp. 431-440, 2008.

[37] R. G. Greenslade and S. N. Mitchell, "Selective action of (-)-2-oxa-4-aminobicyclo[3.1.0] hexane-4,6-dicarboxylate (LY379268), a group II metabotropic glutamate receptor agonist, on basal and phencyclidine-induced dopamine release in the nucleus accumbens shell," Neuropharmacology, vol. 47, no. 1, pp. 1-8, 2004.

[38] P. Seeman, "Glutamate agonists for schizophrenia stimulate dopamine D2High receptors," Schizophrenia Research, vol. 99, no. $1-3$, pp. 373-374, 2008.

[39] P. Seeman and H.-C. Guan, "Phencyclidine and glutamate agonist LY379268 stimulate dopamine D2 high receptors: D2 basis for schizophrenia," Synapse, vol. 62, no. 11, pp. 819-828, 2008.

[40] P. Seeman, G. Battaglia, C. Corti, M. Corsi, and V. Bruno, "Glutamate receptor mGlu2 and mGlu3 knockout striata are dopamine supersensitive, with elevated $\mathrm{D} 2{ }^{\text {High }}$ receptors and marked supersensitivity to the dopamine agonist (+)PHNO," Synapse, vol. 63, no. 3, pp. 247-251, 2009.

[41] P. Seeman, C. Caruso, and M. Lasaga, "Dopamine partial agonist actions of the glutamate receptor agonists LY 354,740 and LY 379,268," Synapse, vol. 62, no. 2, pp. 154-158, 2008.
[42] D. L. Taylor, L. T. Diemel, M. L. Cuzner, and J. M. Pocock, "Activation of group II metabotropic glutamate receptors underlies microglial reactivity and neurotoxicity following stimulation with chromogranin A, a peptide up-regulated in Alzheimer's disease," Journal of Neurochemistry, vol. 82, no. 5, pp. 1179-1191, 2002.

[43] D. L. Taylor, F. Jones, E. S. F. Chen Seho Kubota, and J. M. Pocock, "Stimulation of microglial metabotropic glutamate receptor mGlu2 triggers tumor necrosis factor $\alpha$-induced neurotoxicity in concert with microglial-derived Fas ligand," Journal of Neuroscience, vol. 25, no. 11, pp. 2952-2964, 2005.

[44] P. J. Conn, A. Christopoulos, and C. W. Lindsley, "Allosteric modulators of GPCRs: a novel approach for the treatment of CNS disorders," Nature Reviews Drug Discovery, vol. 8, no. 1, pp. 41-54, 2009.

[45] R. Galici, N. G. Echemendia, A. L. Rodriguez, and P. J. Conn, "A selective allosteric potentiator of metabotropic glutamate (mGlu) 2 receptors has effects similar to an orthosteric mGlu2/3 receptor agonist in mouse models predictive of antipsychotic activity," Journal of Pharmacology and Experimental Therapeutics, vol. 315, no. 3, pp. 1181-1187, 2005.

[46] R. Galici, C. K. Jones, K. Hemstapat, et al., "Biphenylindanone $A$, a positive allosteric modulator of the metabotropic glutamate receptor subtype 2, has antipsychotic- and anxiolytic-like effects in mice," Journal of Pharmacology and Experimental Therapeutics, vol. 318, no. 1, pp. 173-185, 2006.

[47] M. P. Johnson, M. Baez, G. E. Jagdmann Jr., et al., "Discovery of allosteric potentiators for the metabotropic glutamate 2 receptor: synthesis and subtype selectivity of $\mathrm{N}$-(4-(2-methoxyphenoxy)phenyl)-N-(2,2,2-trifluoroethylsulfonyl)pyrid-3- ylmethylamine," Journal of Medicinal Chemistry, vol. 46, no. 15, pp. 3189-3192, 2003. 


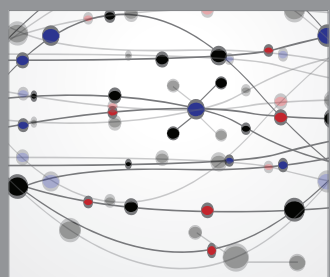

The Scientific World Journal
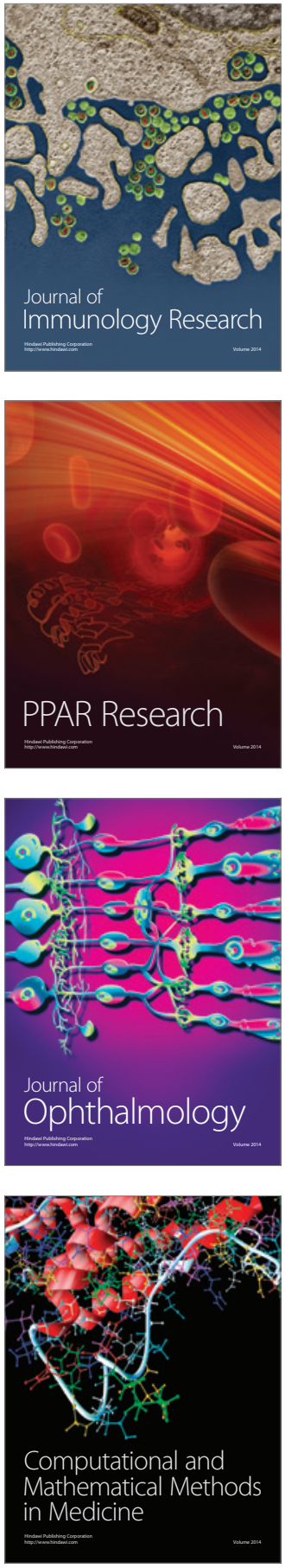

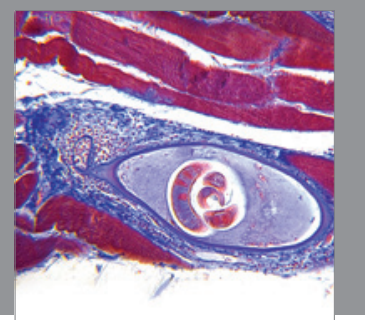

Gastroenterology

Research and Practice
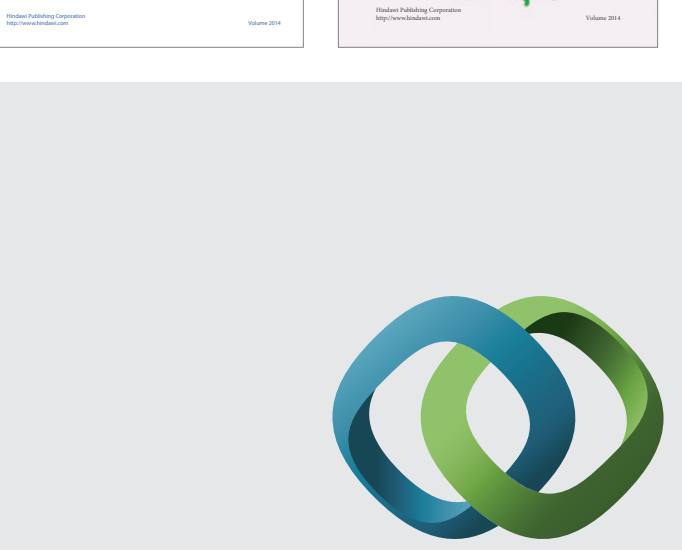

\section{Hindawi}

Submit your manuscripts at

http://www.hindawi.com
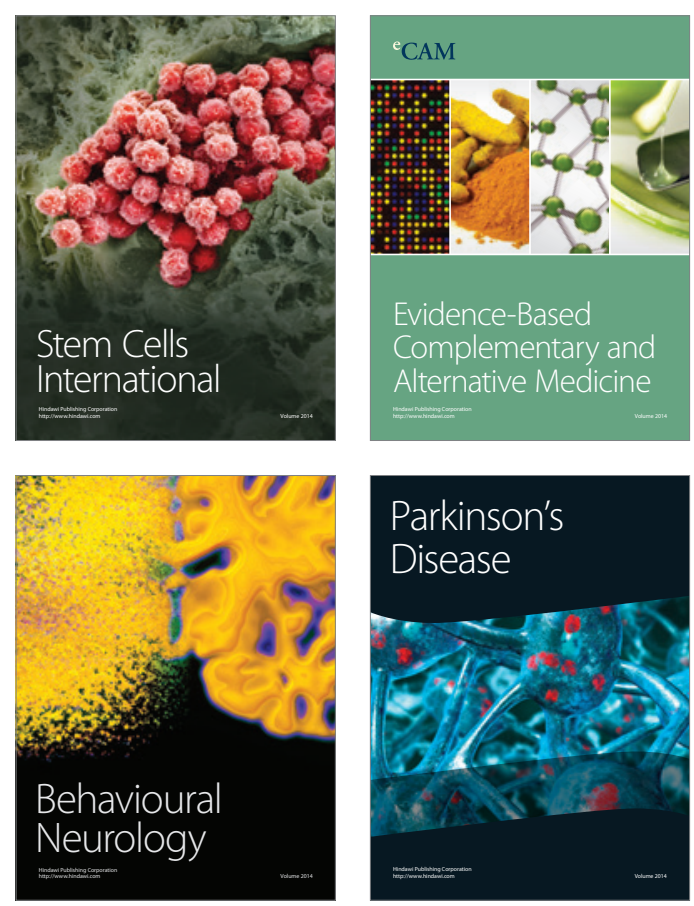

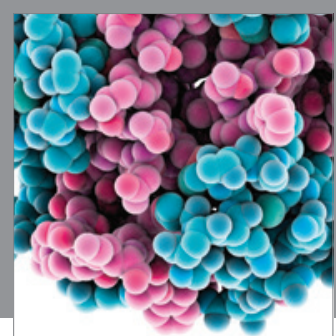

Journal of
Diabetes Research

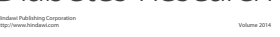

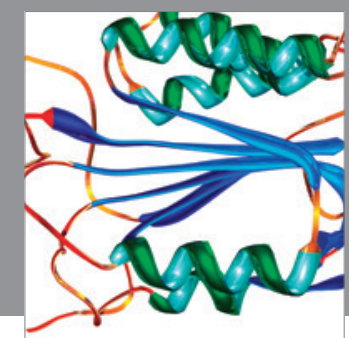

Disease Markers
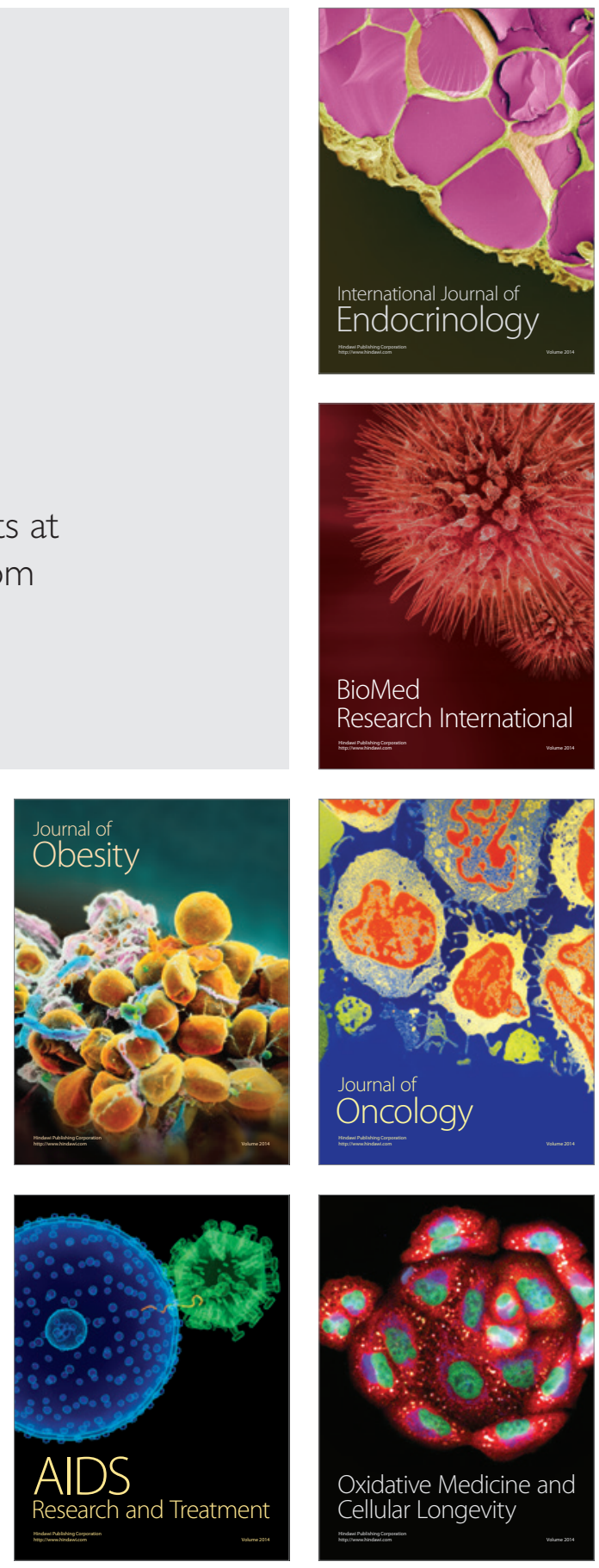